Impact of neutral density fluctuations on gas puff imaging diagnostics

This content has been downloaded from IOPscience. Please scroll down to see the full text.

2017 Nucl. Fusion 57116018

(http://iopscience.iop.org/0029-5515/57/11/116018)

View the table of contents for this issue, or go to the journal homepage for more

Download details:

IP Address: 128.179.252.94

This content was downloaded on 12/08/2017 at 05:48

Please note that terms and conditions apply.

You may also be interested in:

A comparison between a refined two-point model for the limited tokamak SOL and self-consistent plasma turbulence simulations

C Wersal, P Ricci and J Loizu

Plasma shaping effects on tokamak scrape-off layer turbulence

Fabio Riva, Emmanuel Lanti, Sébastien Jolliet et al.

A first-principles self-consistent model of plasma turbulence and kinetic neutral dynamics in the tokamak scrape-off layer

C. Wersal and P. Ricci

Blob properties in full-turbulence simulations of the TCV scrape-off layer

F Nespoli, I Furno, B Labit et al.

Simulations of the effects of density and temperature profile on SMBI penetration depth based on the HL-2A tokamak configuration

Xueke Wu, Huidong Li, Zhanhui Wang et al.

Simulation of plasma turbulence in scrape-off layer conditions: the GBS code, simulation results and code validation

P Ricci, F D Halpern, S Jolliet et al.

High-speed imaging of edge turbulence in NSTX

S.J. Zweben, R.J. Maqueda, D.P. Stotler et al.

Hermes: global plasma edge fluid turbulence simulations

B D Dudson and J Leddy 


\title{
Impact of neutral density fluctuations on gas puff imaging diagnostics
}

\author{
C. Wersal 1 and P. Ricci \\ Ecole Polytechnique Fédérale de Lausanne (EPFL), Swiss Plasma Center (SPC), CH-1015 Lausanne, \\ Switzerland \\ E-mail: christoph.wersal@epfl.ch and paolo.ricci@epfl.ch
}

Received 5 April 2017, revised 23 June 2017

Accepted for publication 5 July 2017

Published 3 August 2017

\begin{abstract}
A three-dimensional turbulence simulation of the SOL and edge regions of a toroidally limited tokamak is carried out. The simulation couples self-consistently the drift-reduced two-fluid Braginskii equations to a kinetic equation for neutral atoms. A diagnostic neutral gas puff on the low-field side midplane is included and the impact of neutral density fluctuations on $D_{\alpha}$ light emission investigated. We find that neutral density fluctuations affect the $D_{\alpha}$ emission. In particular, at a radial distance from the gas puff smaller than the neutral mean free path, neutral density fluctuations are anti-correlated with plasma density, electron temperature, and $D_{\alpha}$ fluctuations. It follows that the neutral fluctuations reduce the $D_{\alpha}$ emission in most of the observed region and, therefore, have to be taken into account when interpreting the amplitude of the $D_{\alpha}$ emission. On the other hand, higher order statistical moments (skewness, kurtosis) and turbulence characteristics (such as correlation length, or the autocorrelation time) are not significantly affected by the neutral fluctuations. At distances from the gas puff larger than the neutral mean free path, a non-local shadowing effect influences the neutral density fluctuations. There, the $D_{\alpha}$ fluctuations are correlated with the neutral density fluctuations, and the high-order statistical moments and measurements of other turbulence properties are strongly affected by the neutral density fluctuations.
\end{abstract}

Keywords: plasma physics, tokamak periphery, turbulence, kinetic neutral atoms, drift-reduced Braginskii, gas-puff imaging, neutral fluctuations

(Some figures may appear in colour only in the online journal)

\section{Introduction}

Understanding the plasma dynamics in the scrape-off layer (SOL) and edge regions of tokamaks is of critical importance to fusion research, since these regions set the boundary conditions for the core plasma, determining the performance of the entire device $[1,2]$. The dynamics in the tokamak periphery results from the interplay and balance of perpendicular and parallel transport, plasma sink at the solid walls, and neutral recycling. Since radial transport is dominated by turbulent transport, measurements of the turbulent dynamics in the SOL and edge regions are of fundamental significance.

Among the different experimental techniques available to study edge and SOL turbulence, we focus here on the gas puff imaging (GPI) diagnostics [3-6], where neutral gas is puffed into the SOL and light emission is recorded by one or more fast cameras with high temporal and spatial resolution. The local light emission stems from atomic processes due to the interaction between the injected neutrals and the plasma, which is optically thin in typical tokamak conditions, so that most emitted photons leave the plasma without further interactions. The cameras measure the integrated local light emission along their lines of sight. The light emission is mostly toroidally localized around the gas puff valve. Therefore, using a fast camera with a tangential view, two-dimensional poloidal snapshots of the local light emission rates are approximately obtained.

While fast cameras can record the whole integrated visible spectrum, single spectral lines are usually selected by optical filters to facilitate the interpretation of the measurements. In particular, the $H_{\alpha}$ line of the Balmer series is often used in hydrogen plasmas (respectively the $D_{\alpha}$ line in deuterium 
plasmas) $[3,5,7,8]$. It corresponds to the transition of an excited hydrogen atom in the third state, $H^{*}(n=3)$, to the second state. The $H^{*}(n=3)$ excited state can originate from various atomic and molecular processes, such as molecular dissociation or electron impact excitation. (An exhaustive list of atomic and molecular processes in hydrogen plasmas can be found in [9].) Due to this large variety of atomic processes and due to the difficulty to separately measure the parameters relevant to the emission process (e.g. plasma density, plasma temperature, atomic and molecular densities of the neutrals), it is not straightforward to interpret the GPI measurements. Assumptions or numerical simulations are necessary.

To interpret GPI measurements, it is often assumed that the emission rate can be estimated by $[4,5,8]$

$$
D_{\alpha}=n n_{\mathrm{n}} r_{\alpha}\left(n, T_{\mathrm{e}}\right),
$$

where $n$ is the electron density (we assume unitary ion charge, $Z=1$, in the present work), $n_{\mathrm{n}}$ is the atomic neutral density, and $r_{\alpha}\left(n, T_{\mathrm{e}}\right)$ is the emission rate coefficient of the $D_{\alpha}$ line, which depends on the electron density and temperature and which is obtained from collisional-radiative modeling (see, e.g. [10]). The contribution of molecular dissociation is therefore neglected in equation (1). Then, two assumptions to interpret GPI images are typically made, i.e. that the structures visible in the light emission are mostly due to the plasma fluctuations, neglecting consequently the fluctuations of the neutral density, and that the plasma density and electron temperature are correlated in the tokamak SOL, which is supported by numerical turbulence simulations [7, 11]. This allows interpreting the GPI measurements and deduce properties of the SOL and edge plasma turbulence, such as spectra, spatial scales, relative fluctuation amplitudes, or blob propagation speed $[3,5,7]$. In order to reduce the number of assumptions, the contribution of plasma and neutral density and electron temperature fluctuations to the light emission have to be measured separately. Experimentally, this is achieved by recording multiple spectral lines for the same lines of sight, taking advantage of the fact that the dependency of the emission rate coefficient, $r\left(n, T_{\mathrm{e}}\right)$, on plasma density and electron temperature can be different for different spectral lines. This was done with helium gas puffs and different spectral He-lines, e.g. for a few lines of sight in the Alcator C-Mod tokamak [3] or, more recently, with two-dimensional images from high-speed cameras and two spectral He-lines in the TJ-II stellarator, which allowed estimating independently plasma density and neutral density fluctuations, showing the impact of neutral density fluctuations on light emission [12].

Despite the progress made on the experimental techniques, because of the complexity of the physics processes involved in the GPI measurements, numerical simulations of the turbulent plasma and the neutral particles are still necessary to confirm the validity of the used assumptions and to guide the interpretation of experimental measurements. Different types of simulations were carried out in the past for this purpose, with various assumptions, taking into account either plasma turbulence, while neglecting neutral fluctuations [7, 8, 11, 13], or simulating neutral density fluctuations, while taking averaged plasma profiles or artificial plasma fluctuations [5, 14-16]. Initial attempts to simulate plasma turbulence and fluctuating neutrals self-consistently were made with two-dimensional plasma simulations and mono-energetic neutrals [17], or with a diffusive neutral model without the back-reaction on the plasma [18].

Our goal is to investigate the impact of neutral density fluctuations on the light emission around the diagnostic gas puff, in particular we focus on the Balmer $D_{\alpha}$ line. For this purpose, we use a three-dimensional turbulence simulation of a limited tokamak SOL and edge, that includes self-consistently plasma turbulence, evolved with a three-dimensional two-fluid driftreduced Braginskii model, and neutral dynamics obtained with a kinetic model. The simulation model does not separate background and fluctuating quantities, allowing for and observing fluctuations of order unity. It also includes two gas puffs around the toroidal rail limiter on the high-field side that fuel the plasma, and a small diagnostic gas puff on the lowfield side equatorial midplane.

This paper is structured as follows. After the Introduction, in section 2 we describe the turbulence simulation that we consider in the present paper and the underlying model. In section 3 , we introduce the synthetic GPI diagnostic and the evaluated $D_{\alpha}$ emission. We describe the influence of neutral fluctuations on the $D_{\alpha}$ emission in section 4 , and discuss it in detail in section 5. The conclusions and an outlook follow.

\section{Self-consistent turbulence simulation}

The simulation presented in this paper is carried out with the GBS code $[19,20]$. GBS evolves plasma density, $n$, electron and ion parallel velocities, $v_{\| \mathrm{e}}$ and $v_{\| \mathrm{i}}$, electron and ion temperatures, $T_{\mathrm{e}}$ and $T_{\mathrm{i}}$, and the generalized vorticity, $\tilde{\omega}$, according to the electrostatic two-fluid drift-reduced Braginskii equations [21, 22] self-consistently with a kinetic model for neutral atoms. The model solved by GBS was derived in $[19,20]$, with the details of the neutral model being described in [23], and it was used to study, amongst other things, the electron temperature drop in front of the limiter in [24]. Herein we briefly summarize the main features of the model and its numerical implementation.

The two-fluid drift reduced Braginskii equations that we consider in the electrostatic limit are

$$
\begin{gathered}
\frac{\partial n}{\partial t}=-\frac{1}{B}[\phi, n]-\nabla_{\|}\left(n v_{\| \mathrm{e}}\right)+\frac{2}{e B}\left[C\left(p_{\mathrm{e}}\right)-e n C(\phi)\right]+\mathcal{D}_{n}(n)+S_{n} \\
+n_{\mathrm{n}} \nu_{\mathrm{iz}}-n \nu_{\mathrm{rec}} \\
\frac{\partial \tilde{\omega}}{\partial t}=-\frac{1}{B}[\phi, \tilde{\omega}]-v_{\| \mathrm{i}} \nabla_{\|} \tilde{\omega}+\frac{B^{2}}{m_{\mathrm{i}} n} \nabla_{\|} j_{\|} \\
\quad+\frac{2 B}{m_{\mathrm{i}} n} C(p)+\mathcal{D}_{\tilde{\omega}}(\tilde{\omega})-\frac{n_{\mathrm{n}}}{n} \nu_{\mathrm{cx}} \tilde{\omega} \\
\frac{\partial v_{\| \mathrm{e}}}{\partial t}=-\frac{1}{B}\left[\phi, v_{\| \mathrm{e}}\right]-v_{\| \mathrm{e}} \nabla_{\|} v_{\| \mathrm{e}}+\frac{e}{\sigma_{\|} m_{\mathrm{e}}} j_{\|} \\
+\frac{e}{m_{\mathrm{e}}} \nabla_{\|} \phi-\frac{T_{\mathrm{e}}}{m_{\mathrm{e}} n} \nabla_{\|} n-\frac{1.71}{m_{\mathrm{e}}} \nabla_{\|} T_{\mathrm{e}}+\mathcal{D}_{v_{\| \mathrm{e}}}\left(v_{\| \mathrm{e}}\right) \\
+\frac{n_{\mathrm{n}}}{n}\left(\nu_{\mathrm{en}}+2 \nu_{\mathrm{iz}}\right)\left(v_{\| \mathrm{n}}-v_{\| \mathrm{e}}\right)
\end{gathered}
$$




$$
\begin{aligned}
\frac{\partial v_{\| \mathrm{i}}}{\partial t}= & -\frac{1}{B}\left[\phi, v_{\| \mathrm{i}}\right]-v_{\| \mathrm{i}} \nabla_{\|} v_{\| \mathrm{i}}-\frac{1}{m_{\mathrm{i}} n} \nabla_{\|} p+\mathcal{D}_{v_{\| \mathrm{i}}}\left(v_{\| \mathrm{i}}\right) \\
& +\frac{n_{\mathrm{n}}}{n}\left(\nu_{\mathrm{iz}}+\nu_{\mathrm{cx}}\right)\left(v_{\| \mathrm{n}}-v_{\| \mathrm{i}}\right) \\
\frac{\partial T_{\mathrm{e}}}{\partial t}= & -\frac{1}{B}\left[\phi, T_{\mathrm{e}}\right]-v_{\| \mathrm{e}} \nabla_{\|} T_{\mathrm{e}} \\
& +\frac{4 T_{\mathrm{e}}}{3 e B}\left[\frac{T_{\mathrm{e}}}{n} C(n)+\frac{7}{2} C\left(T_{\mathrm{e}}\right)-e C(\phi)\right] \\
& +\frac{2 T_{\mathrm{e}}}{3 n}\left[\frac{0.71}{e} \nabla_{\|} j_{\|}-n \nabla_{\|} v_{\| \mathrm{e}}\right] \\
& +\mathcal{D}_{T_{\mathrm{e}}}\left(T_{\mathrm{e}}\right)+\kappa_{\| \mathrm{e}} \nabla_{\|}\left(T_{\mathrm{e}}^{5 / 2} \nabla_{\|} T_{\mathrm{e}}\right)+S_{T_{\mathrm{e}}} \\
& +\frac{n_{\mathrm{n}}}{n} \nu_{\mathrm{iz}}\left[-\frac{2}{3} E_{\mathrm{iz}}-T_{\mathrm{e}}+m_{\mathrm{e}} v_{\| \mathrm{e}}\left(v_{\| \mathrm{e}}-\frac{4}{3} v_{\| \mathrm{n}}\right)\right] \\
& -\frac{n_{\mathrm{n}}}{n} \nu_{\mathrm{en}} m_{\mathrm{e}} \frac{2}{3} v_{\| \mathrm{e}}\left(v_{\| \mathrm{n}}-v_{\| \mathrm{e}}\right)
\end{aligned}
$$

$$
\begin{aligned}
\frac{\partial T_{\mathrm{i}}}{\partial t}= & -\frac{1}{B}\left[\phi, T_{\mathrm{i}}\right]-v_{\| \mathrm{i}} \nabla_{\|} T_{\mathrm{i}} \\
& +\frac{4 T_{\mathrm{i}}}{3 e B}\left[C\left(T_{\mathrm{e}}\right)+\frac{T_{\mathrm{e}}}{n} C(n)-\frac{5}{2} C\left(T_{\mathrm{i}}\right)-e C(\phi)\right] \\
& +\frac{2 T_{\mathrm{i}}}{3 n}\left[\frac{1}{e} \nabla_{\|} j_{\|}-n \nabla_{\|} v_{\| \mathrm{i}}\right]+\mathcal{D}_{T_{\mathrm{i}}}\left(T_{\mathrm{i}}\right)+\kappa_{\| \mathrm{i}} \nabla_{\|}\left(T_{\mathrm{i}}^{5 / 2} \nabla_{\|} T_{\mathrm{i}}\right)+S_{T_{\mathrm{i}}} \\
& +\frac{n_{\mathrm{n}}}{n}\left(\nu_{\mathrm{iz}}+\nu_{\mathrm{cx}}\right)\left[T_{\mathrm{n}}-T_{\mathrm{i}}+\frac{1}{3}\left(v_{\| \mathrm{n}}-v_{\| \mathrm{i}}\right)^{2}\right]
\end{aligned}
$$

with $p=n\left(T_{\mathrm{e}}+T_{\mathrm{i}}\right)$ the total pressure, $j_{\|}=e n\left(v_{\| \mathrm{i}}-v_{\| \mathrm{e}}\right)$ the parallel current, $\kappa_{\| \mathrm{e}}$ and $\kappa_{\| \mathrm{i}}$ the Spitzer heat conduction coefficients, $E_{\mathrm{iz}}$ the effective ionization energy, and $\sigma_{\|}=1.96 e^{2} n \tau_{\mathrm{e}} / m_{\mathrm{e}}$, the parallel conductivity, where $\tau_{\mathrm{e}}$ is the electron collision time. The generalized vorticity, $\tilde{\omega}=\omega+1 / e \nabla_{\perp}^{2} T_{\mathrm{i}}$, is related to the electrostatic potential by $\nabla_{\perp}^{2} \phi=\omega$, where we use the Boussinesq approximation. The following operators are used in the large aspect ratio limit: $\quad \nabla_{\|} A=\hat{\mathbf{b}} \cdot \nabla A, \quad\left[A_{1}, A_{2}\right]=\hat{\mathbf{b}} \cdot\left(\nabla A_{1} \times \nabla A_{2}\right)$, and $C(A)=B / 2[\nabla \times(\hat{\mathbf{b}} / B)] \cdot \nabla A$ with $\hat{\mathbf{b}}=\mathbf{B} / B$. The source terms $\left(S_{n}, S_{T_{\mathrm{e}}}, S_{T_{\mathrm{i}}}\right)$ mimic the outflow of hot plasma from the confined region to the SOL. The small perpendicular diffusion terms $\mathcal{D}_{A}(A)$ are included mostly for numerical reasons. The system is closed by a set of first-principles boundary conditions applied at the magnetic pre-sheath entrance of the limiter plates, derived and discussed in [25]. We remark that at the boundary of the simulation domain towards the main wall, assumed to be at a certain distance from it, we apply vanishing Neumann boundary conditions for $n, v_{\| \mathrm{e}}, v_{\| \mathrm{i}}, T_{\mathrm{e}}$, and $T_{\mathrm{i}}$, and we impose the Dirichlet conditions $\tilde{\omega}=0$ and $\phi=\left\langle\Lambda T_{\mathrm{e}}\right\rangle_{t}$ ( $\Lambda \simeq 3$ and \langle\rangle$_{t}$ is a moving window time average). We use open boundary conditions (vanishing Neumann) for all quantities at the boundary towards the tokamak core.

The interaction of the plasma with the neutral atoms, rigorously deduced from a kinetic description [23], is included through the interaction with the neutral density, $n_{\mathrm{n}}$, parallel velocity, $v_{\| \mathrm{n}}$, and temperature, $T_{\mathrm{n}}$. These moments of the neutral distribution function are obtained from the solution of the kinetic neutral equation for a mono-atomic neutral species $\frac{\partial f_{\mathrm{n}}}{\partial t}+\mathbf{v} \cdot \frac{\partial f_{\mathrm{n}}}{\partial \mathbf{x}}=-\nu_{\mathrm{iz}} f_{\mathrm{n}}-\nu_{\mathrm{cx}}\left(f_{\mathrm{n}}-\frac{n_{\mathrm{n}}}{n_{\mathrm{i}}} f_{\mathrm{i}}\right)+\nu_{\mathrm{rec}} f_{\mathrm{i}}$

where $f_{\mathrm{n}}$ and $f_{\mathrm{i}}$ are the neutral and ion distribution functions. The ionization, charge-exchange, and recombination processes as well as the elastic electron-neutral collisions are described, respectively, through the use of Krook operators with collision frequencies defined as $\nu_{\mathrm{iz}}=n_{\mathrm{e}}\left\langle v_{\mathrm{e}} \sigma_{\mathrm{iz}}\left(v_{\mathrm{e}}\right)\right\rangle, \nu_{\text {rec }}=n_{\mathrm{e}}\left\langle v_{\mathrm{e}} \sigma_{\text {rec }}\left(v_{\mathrm{e}}\right)\right\rangle, \nu_{\mathrm{cx}}=n_{\mathrm{i}}\left\langle v_{\mathrm{i}} \sigma_{\mathrm{cx}}\left(v_{\mathrm{i}}\right)\right\rangle$, and $\nu_{\mathrm{en}}=n_{\mathrm{e}}\left\langle v_{\mathrm{e}} \sigma_{\mathrm{en}}\left(v_{\mathrm{e}}\right)\right\rangle$, where $\sigma_{\mathrm{iz}}, \sigma_{\mathrm{rec}}, \sigma_{\mathrm{cx}}$, and $\sigma_{\mathrm{en}}$ are the ionization, recombination, charge-exchange, and elastic electron-neutral cross-sections. (The $\langle\cdot\rangle$ operator denotes the averaging over the Maxwellian electron, or ion, distribution function.) We note that we neglected the electron-neutral collisions in equation (8) due to the high electron to neutral mass ratio [23]. The neutral model is chosen to be as simple as possible, while still keeping the main plasma-neutral interactions with a kinetic neutral description to allow for simulations in short and long neutral mean free path scenarios. Although molecular processes become important at low temperatures $\left(T_{\mathrm{e}} \lesssim 10 \mathrm{eV}\right.$ ), especially for quantitative comparison with experiments, they are neglected in the current model to facilitate the interpretation and understanding of the simulation results.

Equations (2)-(7) are solved by the GBS code using a second order finite difference scheme, except for the $\left[A_{1}, A_{2}\right]$ operators that are discretized by using the Arakawa scheme [26]. Time integration is carried out with the standard 4th order Runge-Kutta method [27]. The correctness of the implementation and numerical discretization of equations (2)-(7) in the GBS code was rigorously verified with the method of manufactured solutions [28]. The solution of equation (8) is obtained in the limit of $\tau_{\mathrm{n}}<\tau_{\text {turb }}$ ( $\tau_{\mathrm{n}}$ is the mean flight time of a neutral atom, $\tau_{\text {turb }}$ is the turbulent timescale) and $1 / k_{\|}>\lambda_{\mathrm{mfp}, \mathrm{n}}\left(\lambda_{\mathrm{mfp}, \mathrm{n}}\right.$ is the mean free path of the neutrals) by using the method of characteristics [23].

The simulation used for the present study considers a limited tokamak with a toroidal rail limiter on the high-field side equatorial midplane and circular flux surfaces. The simulation includes the SOL and part of the edge region, where the magnetic field lines lie on closed flux surfaces without intersecting the wall, similarly to [29]. (We remark that we present here for the first time a GBS simulation that includes the SOL and edge regions, as well as the self-consistent neutral-plasma interaction terms.) The normalized dimensions of the simulated tokamak are $R_{0}=500 \rho_{\mathrm{s} 0}$ and $a_{0}=800 \rho_{\mathrm{s} 0} / 2 \pi, R_{0}$ and $a_{0}$ being the major and minor radii, $\rho_{\mathrm{s} 0}=c_{\mathrm{s} 0} / \Omega_{\mathrm{ci}}, \Omega_{\mathrm{ci}}=e B_{0} / m_{\mathrm{i}}$, and $c_{\mathrm{s} 0}=\sqrt{T_{\mathrm{e} 0} / m_{\mathrm{i}}}$. The poloidal direction is described by the coordinate $y$, with $y=0$ at the lower side of the limiter, $y=400 \rho_{\mathrm{s} 0}$ at the low-field side equatorial midplane, and $y=800 \rho_{\mathrm{s} 0}$ at the upper side of the limiter. The radial extents of the simulated SOL and edge regions are $75 \rho_{\mathrm{s} 0}$ each and the separatrix is located at $r=0$. The resistivity, normalized to $R_{0} / c_{\mathrm{s} 0}$, is defined as $\tilde{\nu}=\tilde{\nu}_{0}\left(T_{\mathrm{e}} / T_{\mathrm{e} 0}\right)^{-3 / 2}$, taking into account the Spitzer dependency and being $\tilde{\nu}_{0}=R_{0} m_{\mathrm{e}} /\left(1.96 c_{\mathrm{s} 0} m_{\mathrm{i}} \tau_{\mathrm{e}}\right)$. The dimensionless parallel electron heat conductivity is $\tilde{\kappa}_{\| \mathrm{e}}=3.16 \times 2 T_{\mathrm{e} 0} \tau_{\mathrm{e}} /\left(3 m_{\mathrm{e}} c_{\mathrm{s} 0} R_{0}\right)$ 
and the dimensionless parallel ion heat conductivity is $\tilde{\kappa}_{\| \mathrm{i}}=3.9 \times 2 T_{\mathrm{i} 0} \tau_{\mathrm{i}} /\left(3 m_{\mathrm{i}} c_{\mathrm{s} 0} R_{0}\right)$. With the normalization parameters $n_{0}=2 \cdot 10^{19} \mathrm{~m}^{-3}, T_{\mathrm{e} 0}=T_{\mathrm{i} 0}=20 \mathrm{eV}$, and $B_{0}=$ $0.5 \mathrm{~T}$, it results $\rho_{\mathrm{s} 0} \simeq 0.9 \mathrm{~mm}, R_{0} \simeq 44 \mathrm{~cm}, a_{0} \simeq 11 \mathrm{~cm}$, and $R_{0} / c_{\mathrm{s} 0} \simeq 10 \mu \mathrm{s}$. The normalized values for the resistivity and the parallel heat conductivities calculated from the parameters above $\left(\tilde{\nu}=0.03, \tilde{\kappa}_{\| \mathrm{e}}=39.6\right.$, and $\left.\tilde{\kappa}_{\| \mathrm{i}}=1.1\right)$ are modified to $\tilde{\nu}=0.1, \tilde{\kappa}_{\| \mathrm{e}}=10$, and $\tilde{\kappa}_{\| \mathrm{i}}=0.5$ to reduce the numerical cost of the simulation. The ion to electron mass ratio is set to $m_{\mathrm{i}} / m_{\mathrm{e}}=200$ for the same reason. We also impose $E_{\mathrm{iz}}=30$ $\mathrm{eV}$. We note that recombination processes can be neglected at $T_{\mathrm{e} 0}=20 \mathrm{eV}$ since $\nu_{\mathrm{rec}} / \nu_{\mathrm{iz}} \approx 10^{-6}$ (within our simple atomic neutral model this assumption holds for $T_{\mathrm{e}} \gtrsim 2 \mathrm{eV}$, corresponding to $\nu_{\mathrm{rec}} / \nu_{\mathrm{iz}} \lesssim 10^{-2}$ ). The numerical grid used for solving the plasma drift-reduced Braginskii equations is $191 \times 495 \times 64$ (radial, poloidal, toroidal) and the grid for the kinetic neutral equation is $24 \times 360$ (radial, poloidal), for each of the 64 poloidal planes.

Since there is no separation between equilibrium and fluctuating quantities in the drift-reduced Braginskii equations, equations (2)-(7), the plasma profiles result selfconsistently from the interplay between perpendicular and parallel transport, losses at the sheath, ionization processes, and heat outflowing from the tokamak core to the edge region. The latter is mimicked by the temperature source terms, $S_{T_{\mathrm{e}}}$ and $S_{T_{\mathrm{i}}}$, which are constant in time, poloidally uniform, and radially Gaussian with a width of $5 \rho_{\mathrm{s} 0}$ centered around $r_{\mathrm{s}}=-75 \rho_{\mathrm{s} 0}$, i.e. the location of the boundary of the simulated domain towards the tokamak core. In the present simulation it is assumed that the source of plasma density is solely due to ionization of neutral atoms in the simulated volume, therefore the plasma density source $S_{n}$ in equation (2), mimicking the outflow of plasma density from the core, vanishes. The plasma flowing along the magnetic field lines and arriving at the limiter plates is recycled, assuming that $80 \%$ is reflected specularly and $20 \%$ is absorbed on the surface and released with a thermal distribution of $3 \mathrm{eV}$, i.e. approximately the energy of neutral atoms after Frank-Condon dissociation $[9,30]$. We assume the same reflection coefficient for neutrals arriving at the limiter.

To fuel the plasma and compensate for the radial plasma losses, two gas puffs on the high-field side, above and below the limiter, and a constant inflow of neutrals from the main wall (mimicking main wall recycling) are included in the simulation. The two gas puffs are toroidally constant and, together, account for approximately $40 \%$ of the ionization. The small constant inflow of neutrals from the main wall accounts for approximately $15 \%$ of the total ionization and the recycling at the limiter for approximately $40 \%$. (The remaining $5 \%$ is due to the diagnostics gas puff described in section 3.)

The gas puff inlets lie outside the simulated domain, since its outer boundary (the boundary towards the main vessel wall) does not coincide with the physical wall. Therefore, we assume that the hydrogen molecules from the gas puff are dissociated into atoms and somewhat diffuse before entering the simulated domain. We account for this by setting the inflowing distribution function at the outer boundary, $\mathbf{x}_{\mathrm{b}}$, to

$$
f_{\mathrm{n}, \mathrm{gp}}\left(\mathbf{x}_{\mathrm{b}}, \mathbf{v}\right) \propto \exp \left(-\frac{\left(y_{\mathrm{b}}-y_{0 \mathrm{gp}}\right)^{2}}{2 \Delta y_{\mathrm{gp}}^{2}}\right) \chi_{\mathrm{in}}\left(\mathbf{x}_{\mathrm{b}}, \mathbf{v}\right),
$$

where $y_{\mathrm{b}}$ is the outer boundary poloidal coordinate $\left(y_{\mathrm{b}}=0\right.$ at the high-field side equatorial midplane), $y_{0 \mathrm{gp}}= \pm 40 \rho_{\mathrm{s} 0}$ are the locations and $y_{\mathrm{gp}}=20 \rho_{\mathrm{s} 0}$ the width of the gas puffs. The inflowing velocity distribution, $\chi_{\mathrm{in}}$, is assumed to be

$$
\chi_{\text {in }}\left(\mathbf{x}_{\mathrm{b}}, \mathbf{v}\right)=\frac{3}{4 \pi} \frac{m^{2}}{T_{\mathrm{b}}^{2}} \cos (\theta) \exp \left(-\frac{m v^{2}}{2 T_{\mathrm{b}}}\right)
$$

being $\theta=\arccos (\hat{\boldsymbol{\Omega}} \cdot \hat{\mathbf{n}}), \hat{\mathbf{\Omega}}=\mathbf{v} / v, \hat{\mathbf{n}}$ the normal vector perpendicular to the boundary and pointing into the plasma region, and $T_{\mathrm{b}}=3 \mathrm{eV}$.

After a transient, a quasi-steady state is achieved in our simulation where sources and losses balance and the total amount of particles in the system is approximately constant. Our investigation focuses on this quasi-steady state. Poloidal snapshots of plasma density, electron temperature, neutral density, and ionization source, $S_{\mathrm{iz}}$, are shown in figure 1 . We observe strong poloidal asymmetries, particularly visible for the plasma density in the edge region, which is higher on the high-field side than on the low-field side, and in the electron and ion temperatures, which are higher on the low-field side than on the high-field side. In the SOL, the electron temperature drop towards the limiter is due to the balance between radial turbulent transport, parallel heat conduction and convection, the ionization energy loss close to the limiter, and the parallel outflow of plasma to the limiter (see, e.g. our recent work on a refined two-point model [24]).

Time-averaged radial profiles of plasma density, electron temperature, and neutral density on the low-field side equatorial midplane $\left(350 \rho_{\mathrm{s} 0}<y<450 \rho_{\mathrm{s} 0}\right)$, where the subsequent investigations on plasma and neutral fluctuations are performed, are shown in figure 2 . The neutral density decays approximately exponentially with a scale length of $60 \rho_{\mathrm{s} 0}$ from the outer domain boundary, resulting from ionization and charge exchange processes. We define this scale length as the effective mean free path of the neutrals, $\lambda_{\mathrm{mfp}, \mathrm{n}}$. On the other hand, the plasma density decays from the core to the vessel wall with a scale length comparable to the radial domain size, approximately $180 \rho_{\mathrm{s} 0}$. The electron temperature has a short decay length in the closed flux-surface region closest to the core (approximately $20-30 \rho_{\mathrm{s} 0}$ ), and a decay length comparable to the one of the density in the SOL.

\section{The GPI synthetic diagnostics}

In addition to the aforementioned two gas puffs on the highfield side, a small diagnostic gas puff is placed on the lowfield side equatorial midplane. To simplify the geometry of our simulation, we assume that the diagnostic gas puff is toroidally constant. Moreover, we impose that it injects neutrals with the same distribution function as the two fueling gas puffs, equation (9), but with lower amplitude. This diagnostic gas puff does not contribute significantly to the plasma fueling (it accounts for approximately $5 \%$ of the ionization in the 

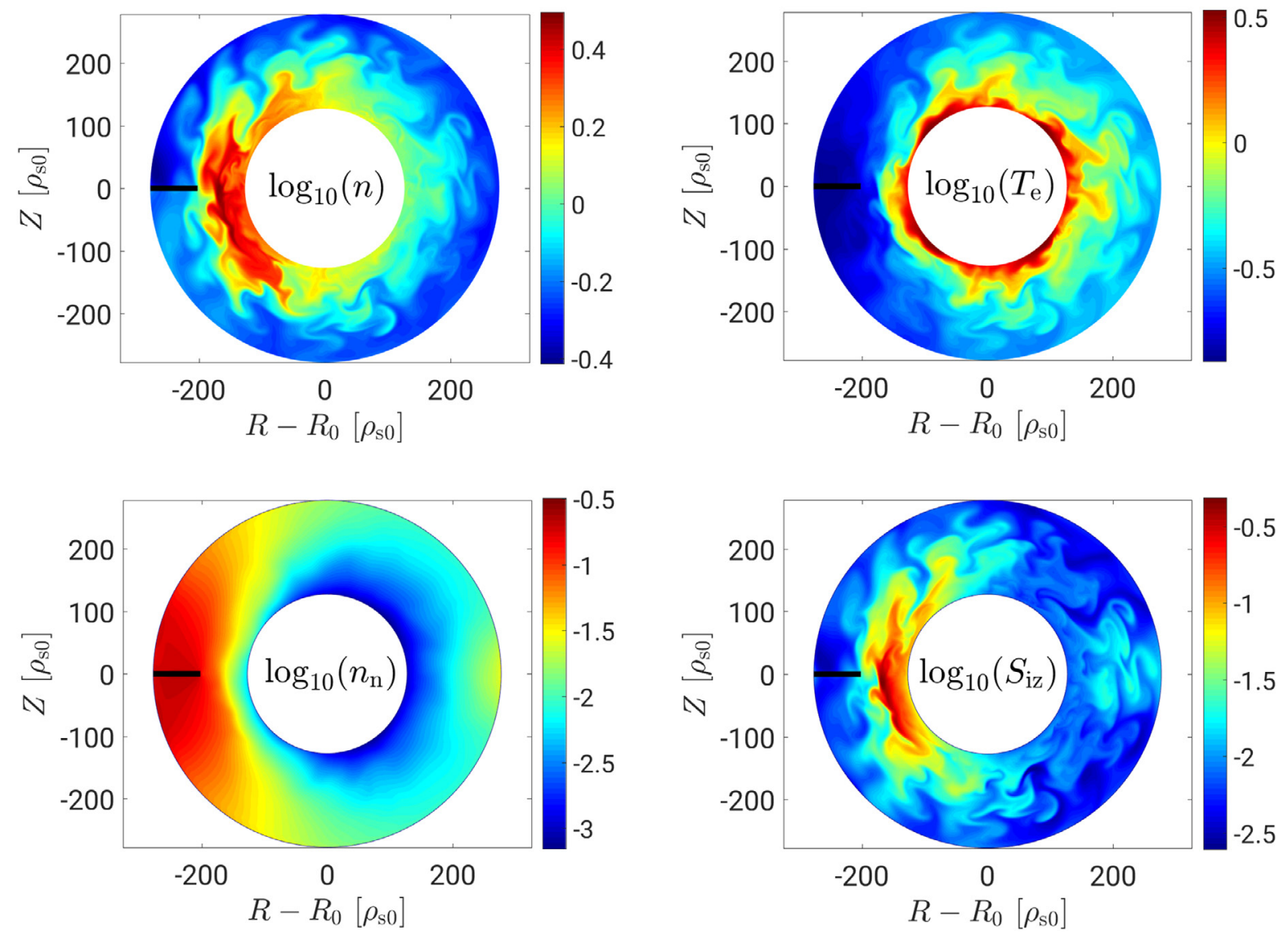

Figure 1. Poloidal snapshots of plasma density, electron temperature, neutral density, and ionization rate, for the simulation considered in the present paper. The densities are normalized to $n_{0}$, the electron temperature to $T_{0}$, and the ionization rate to $n_{0} c_{\mathrm{s} 0} / R_{0}$.

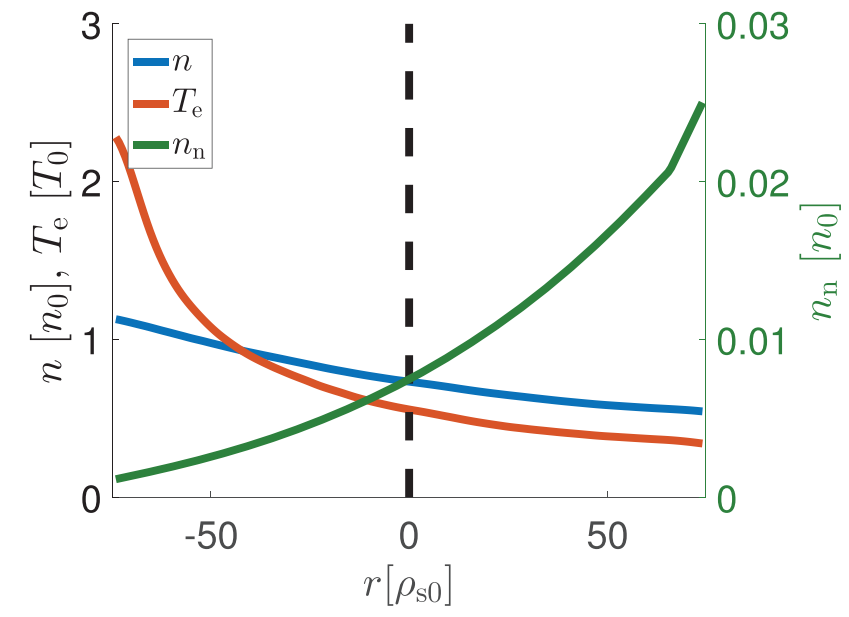

Figure 2. Radial profiles of plasma density, electron temperature, and neutral density on the low-field side equatorial midplane, time, toroidally, and poloidally $\left(350 \rho_{\mathrm{s} 0}<y<450 \rho_{\mathrm{s} 0}\right)$ averaged.

simulation), as we also verified by turning it off. The same can also be inferred from figure 1 , where one can observe that the ionization rate, $S_{\mathrm{iz}}$, is rather small at the low-field side around the diagnostic gas puff. We note that toroidally constant gas puffs are atypical in experiments. However, our results are not significantly affected by the geometrical details of the gas puff since, in the present work, we do not address the issue of the integration of the signal along the lines of sight of a camera,

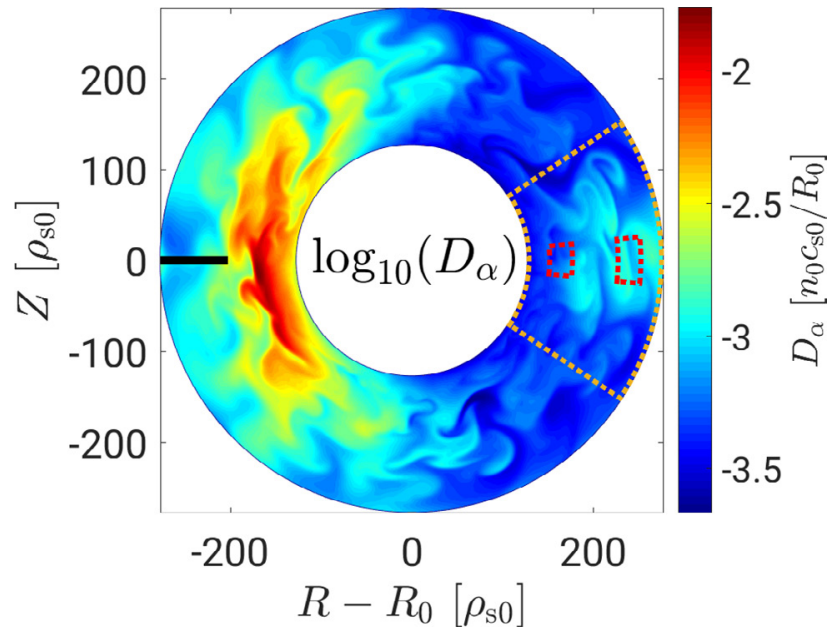

Figure 3. Poloidal snapshot of the $D_{\alpha}$ emission rate. The large yellow dotted area indicates the spatial region shown in figures 4 , 5 and 11 . The smaller red dotted areas indicate the spatial regions where the joint probability distributions shown in figures 8 and 9 are calculated.

for which a toroidally localized gas puff is needed, but we analyze the local light emission.

We focus on the local emission rate of the Balmer $D_{\alpha}$ line, which we calculate as

$$
D_{\alpha}=n_{e} n_{n} r_{D_{\alpha}}\left(n_{e}, T_{e}\right),
$$



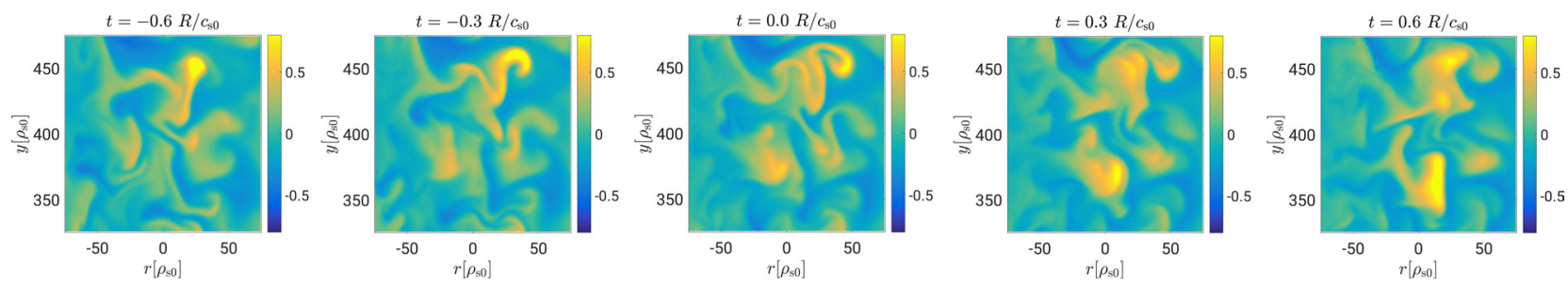

Figure 4. Five snapshots, separated by $0.3 R_{0} / c_{\mathrm{s} 0} \simeq 3 \mu \mathrm{s}$, representing normalized fluctuations of $D_{\alpha}$ light emission, $\left(D_{\alpha}-\left\langle D_{\alpha}\right\rangle\right) /\left\langle D_{\alpha}\right\rangle$, in front of the gas puff. The considered spatial domain is indicated by a yellow dotted contour in figure 3 . The LCFS is located at $r=0$ and the low-field side equatorial midplane is at $y=400 \rho_{\mathrm{s} 0}$. The snapshots in figures 1, 3,5 and 11 are evaluated at $t=0$.
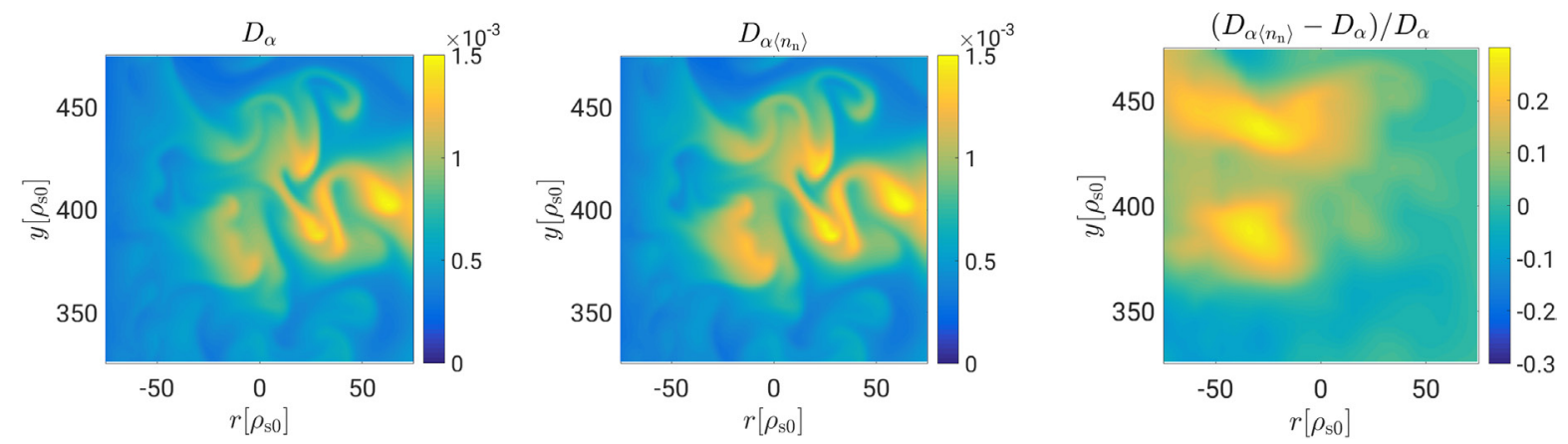

Figure 5. Snapshots of $D_{\alpha}$ emission (left) and $D_{\alpha\left\langle n_{\mathrm{n}}\right\rangle}$ emission, evaluated by removing the contribution of neutral density fluctuations (middle), and their relative difference (right).
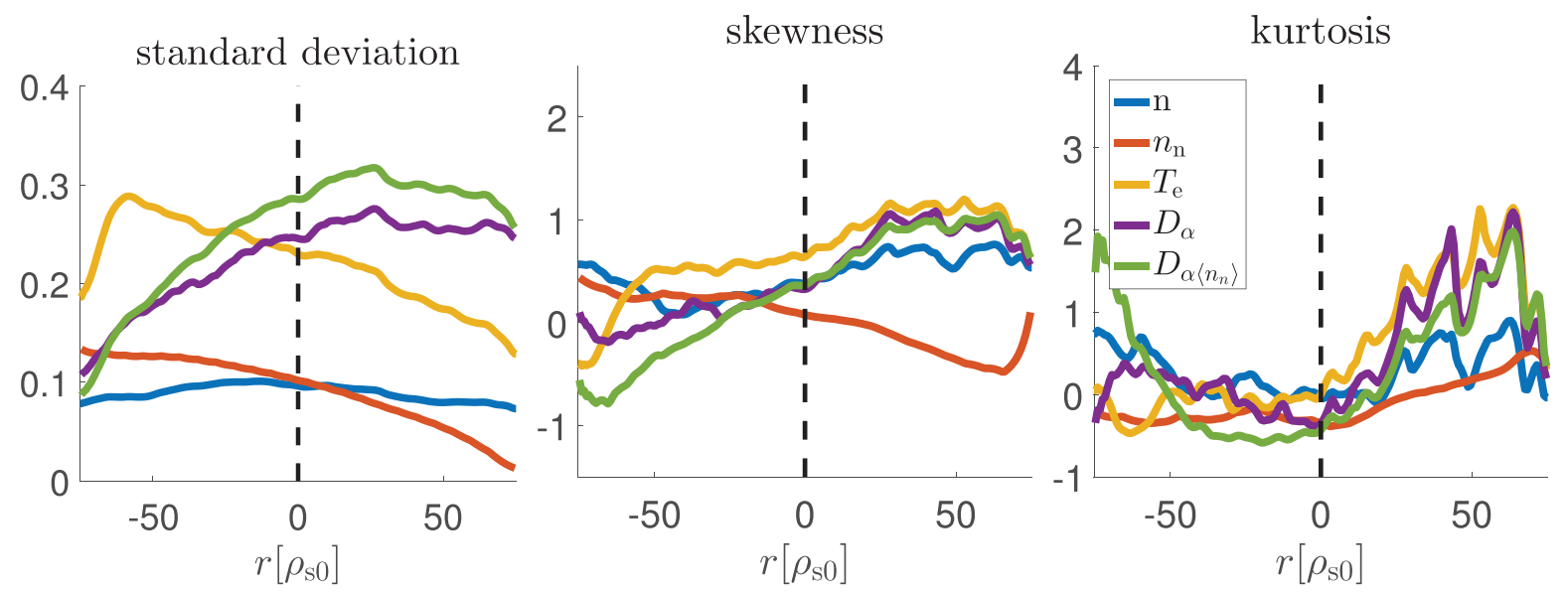

Figure 6. Radial profiles of standard deviation (left), skewness (middle), and kurtosis (right) of normalized plasma and neutral density, electron temperature, and $D_{\alpha}$ fluctuations with and without neutral density fluctuations at the low-field side midplane.

where $r_{D_{\alpha}}$ is the emission coefficient that depends on electron density and temperature [10] as tabulated in the OpenADAS database $^{1}$. This emission coefficient is used throughout this paper. A snapshot of the $D_{\alpha}$ emission rate is shown in figure 3 on the full poloidal cross-section. Figure 4 displays the temporal evolution of the normalized $D_{\alpha}$ fluctuations in the region in front of the diagnostic gas puff around the low-field side equatorial midplane. (This region is indicated by a yellow dotted contour in figure 3.) Normalized $D_{\alpha}$ emission fluctuations are defined as $\left(D_{\alpha}-\left\langle D_{\alpha}\right\rangle\right) /\left\langle D_{\alpha}\right\rangle$, where $\left\langle D_{\alpha}\right\rangle$ denotes the toroidal and time average of $D_{\alpha}$. We note that the toroidal average can be performed since an axisymmetric system is

\footnotetext{
${ }^{1}$ OpenADAS—http://open.adas.ac.uk
}

investigated, and the time average is taken over a time window of $\Delta t=40 R_{0} / c_{\mathrm{s} 0}$ that covers several fluctuation times during the quasi-steady state phase of the simulation. Similar definitions apply to other quantities.

\section{Impact of neutral fluctuations on GPI}

The neutral density in the SOL and edge regions is not easily measured experimentally. It is therefore difficult to disentangle the contributions of plasma density, electron temperature, and neutral density fluctuations to the fluctuations of the $D_{\alpha}$ emission, equation (11). To investigate the impact of neutral density fluctuations on the $D_{\alpha}$ emission, we evaluate it by using the averaged neutral density: 

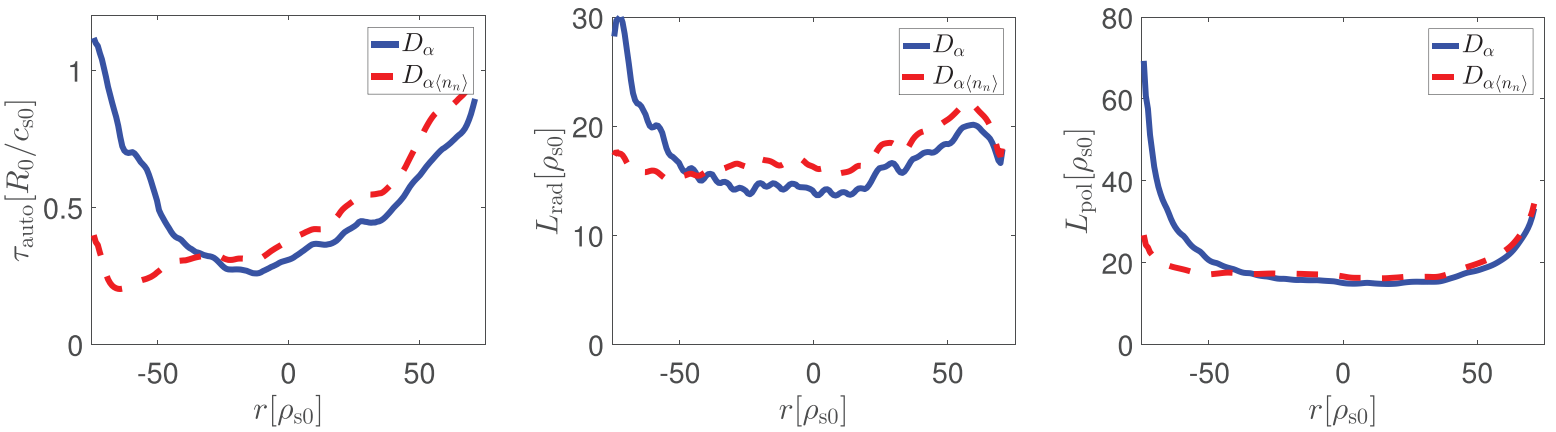

Figure 7. Radial dependence of autocorrelation time (left panel) and correlation lengths along the radial (middle panel) and poloidal (right panel) directions for the $D_{\alpha}$ emission with and without neutral fluctuations.
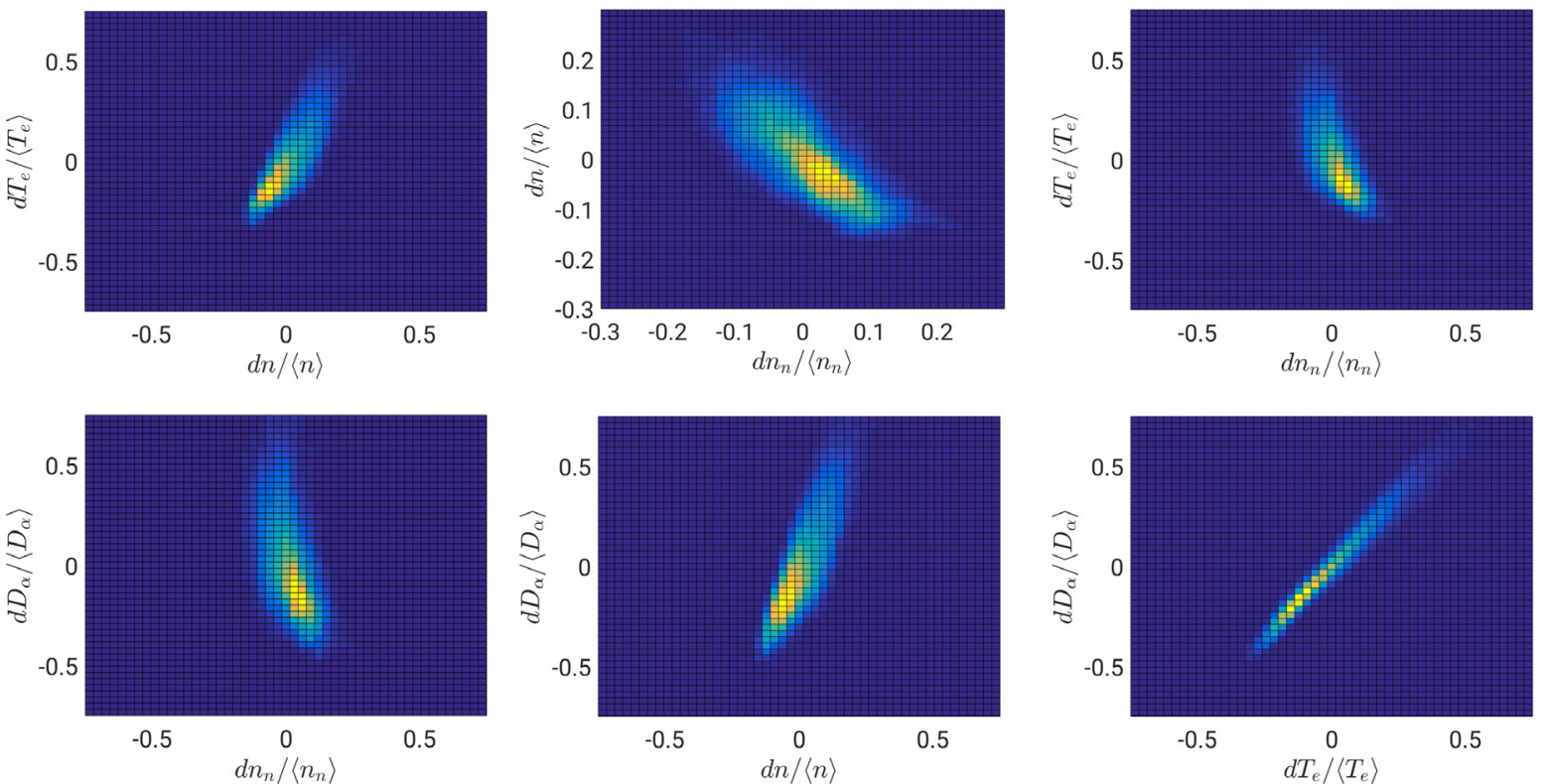

Figure 8. Joint probability distribution between fluctuations of plasma density, electron temperature, neutral density, and $D_{\alpha}$ emission in front of the low-field side diagnostic gas puff in the $\operatorname{SOL}\left(25 \rho_{\mathrm{s} 0}\right.$ by $25 \rho_{\mathrm{s} 0}$ window, centered around $r=37.5 \rho_{\mathrm{s} 0}$ and $y=400 \rho_{\mathrm{s} 0}$, right red dotted region in figure 3 ).

$$
D_{\alpha\left\langle n_{\mathrm{n}}\right\rangle}=n_{\mathrm{e}}\left\langle n_{\mathrm{n}}\right\rangle r_{D_{\alpha}}\left(n_{\mathrm{e}}, T_{\mathrm{e}}\right),
$$

removing thereby the neutral density fluctuations, while keeping plasma density and electron temperature fluctuations.

We show $D_{\alpha}$ and $D_{\alpha\left\langle n_{\mathrm{n}}\right\rangle}$ in figure 5 (left and middle panels) and their normalized difference in figure 5 (right panel). While $D_{\alpha}$ and $D_{\alpha\left\langle n_{\mathrm{n}}\right\rangle}$ show similar spatial patterns, neglecting neutral density fluctuations leads to errors of the order of $30 \%$ in the intensity of the light emission, in particular in the closed flux-surface region. This therefore shows that the local $D_{\alpha}$ emission amplitude might be significantly influenced by neutral fluctuations.

To investigate the impact of neutral fluctuations on other typical quantities obtained from GPI diagnostics, we evaluate the standard deviation, the skewness, the kurtosis, the autocorrelation time ( $\tau_{\text {auto }}$ ), and the radial and poloidal correlation lengths $\left(L_{\mathrm{rad}}\right.$ and $\left.L_{\mathrm{pol}}\right)$ from the normalized $D_{\alpha}$ and $D_{\alpha\left\langle n_{\mathrm{n}}\right\rangle}$ fluctuations, respectively. These are shown in figures 6 and 7 .
The standard deviation of the normalized $D_{\alpha\left\langle n_{\mathrm{n}}\right\rangle}$ emission in figure 6 (left panel) is up to $20 \%$ larger than the standard deviation of the $D_{\alpha}$ emission in the SOL and their difference decreases when approaching the core. In addition, in the SOL we also observe that $D_{\alpha}$ fluctuations are larger than $n, n_{n}$, and $T_{e}$ fluctuations, displaying also a different radial dependence. The skewness of the turbulent fields is analysed in the middle panel of figure 6 . We note that the skewness of $D_{\alpha}$ and $D_{\alpha\left\langle n_{n}\right\rangle}$ are very similar in the SOL, and both of them follow the skewness of plasma density and electron temperature and increase radially. On the other hand, we observe a difference between the $D_{\alpha}$ and $D_{\alpha\left\langle n_{\mathrm{n}}\right\rangle}$ skewness in the confined region, where they are also quite different with respect to the skewness of plasma density and electron temperature. The skewness of the neutral density decreases radially and is negative in the SOL and positive in the edge. Similar remarks can be made for the kurtosis, figure 6 (right panel), namely, $D_{\alpha}$ and $D_{\alpha\left\langle n_{\mathrm{n}}\right\rangle}$ show similar behavior in the SOL region, and a discrepancy in the confined region. 

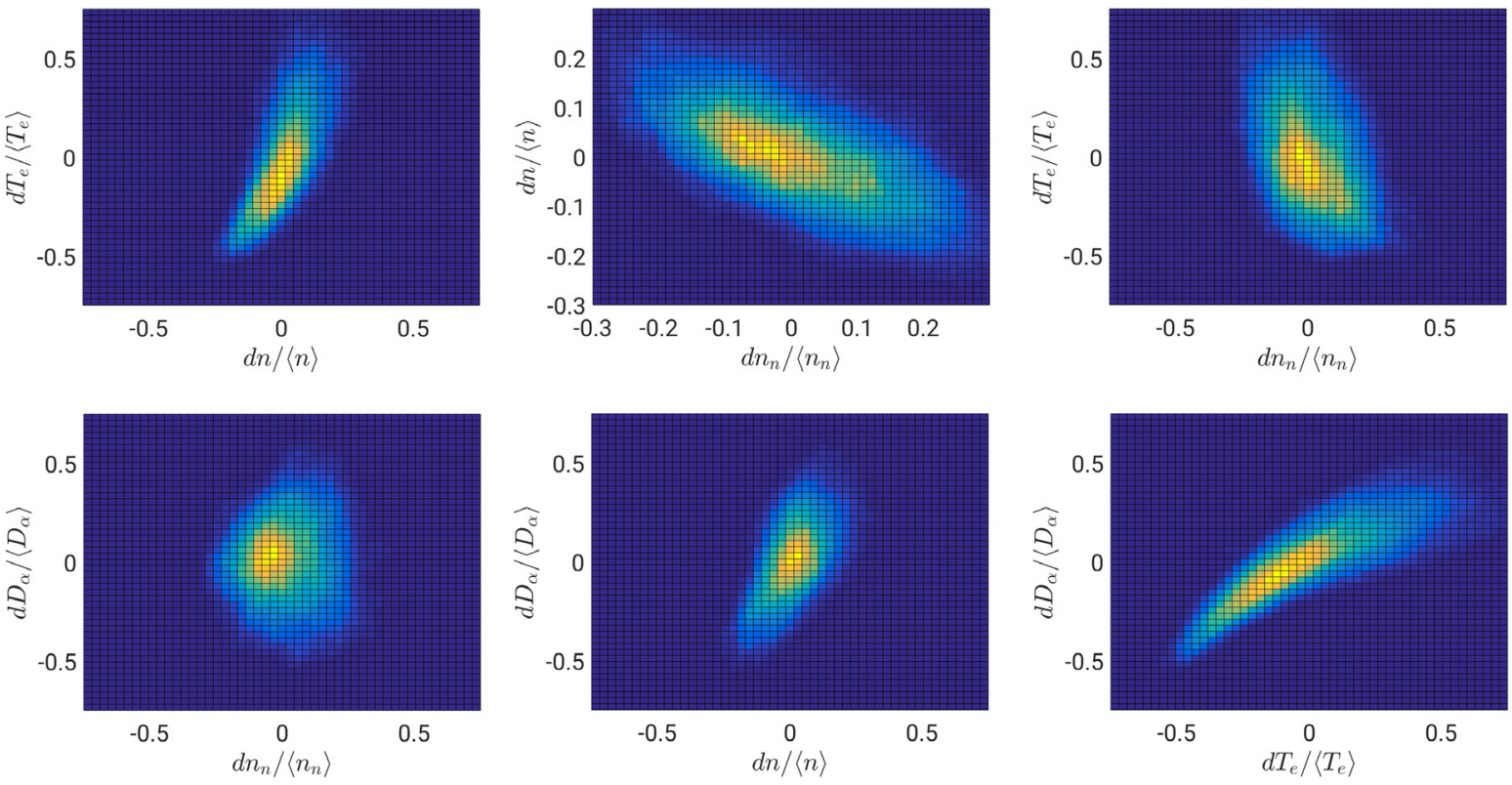

Figure 9. Same plots as in figure 8 in the edge $\left(25 \rho_{\mathrm{s} 0}\right.$ by $25 \rho_{\mathrm{s} 0}$ window, centered around $r=-37.5 \rho_{\mathrm{s} 0}$ and $y=400 \rho_{\mathrm{s} 0}$, left red dotted region in figure 3 ).

To evaluate $\tau_{\text {auto }}, L_{\text {rad }}$, and $L_{\text {pol }}$, we use the definitions in [7] and [8], i.e.

$$
L=1.66 \frac{\delta}{\sqrt{-\ln C_{i j}}}
$$

and

$$
C_{i i}\left(\tau_{\text {auto }}\right)=\frac{1}{2},
$$

where $\delta$ is the distance between the two positions $i$ and $j$ (corresponding experimentally to two lines of sight), and $C_{i j}$ is the zero-time-delay cross-correlation function between the signals obtained from these two positions. For our analysis we choose $\delta=3 \rho_{\mathrm{s} 0}$ (the results do not depend on the choice of $\delta$ for $\left.1.5 \rho_{\mathrm{s} 0} \lesssim \delta \lesssim 10 \rho_{\mathrm{s} 0}\right)$.

Radial profiles of $\tau_{\text {auto }}, L_{\mathrm{rad}}$, and $L_{\mathrm{pol}}$ are shown in figure 7 . Neutral density fluctuations do not have a large impact on these measurements in the SOL. On the other hand, an effect of neutral density fluctuations can be observed in the confined region towards the core, similarly to what is observed for the statistical moments in figure 6 . We note that converting normalized to dimensional units reveals $\tau_{\text {auto }} \approx 3-8 \mu \mathrm{s}$, $L_{\mathrm{rad}} \approx 1.5-2 \mathrm{~cm}$, and $L_{\mathrm{pol}} \approx 2 \mathrm{~cm}$, values that are similar to the ones found in the C-Mod tokamak [7, 8].

\section{Discussion}

We analyze the link between neutral and plasma fluctuations to explain the impact of neutral density fluctuations on the $D_{\alpha}$ emission rate. We first study the correlations and anticorrelations between $n, n_{\mathrm{n}}, T_{\mathrm{e}}$, and $D_{\alpha}$ through their joint probability distributions, evaluated as the joint histograms of their normalized fluctuations. These are presented for the SOL $\left(25 \rho_{\mathrm{s} 0}<r<50 \rho_{\mathrm{s} 0}\right)$ in figure 8 , and for the edge

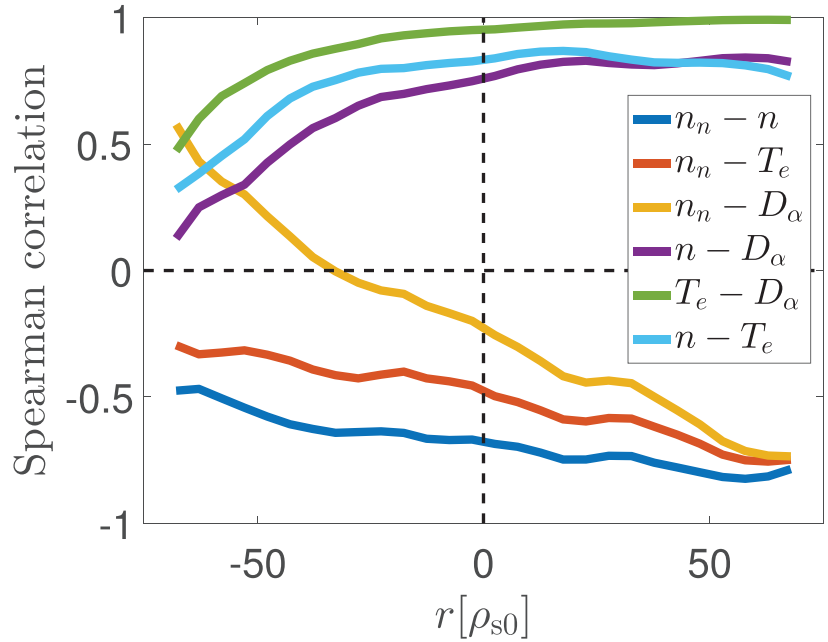

Figure 10. Radial profiles of the Spearman correlation coefficient between plasma and neutral density, electron temperature, and $D_{\alpha}$ emission.

$\left(-50 \rho_{\mathrm{s} 0}<r<-25 \rho_{\mathrm{s} 0}\right)$ in figure 9. Both regions (SOL and edge) show that plasma density and electron temperature fluctuations are correlated (an observation made also in other SOL turbulence simulations $[7,11])$. On the other hand, the neutral density is anti-correlated with both plasma density and electron temperature, particularly in the SOL. The anti-correlation between $D_{\alpha}$ emission and neutral density, observed in the SOL, disappears in the confined region, and the correlation between $D_{\alpha}$ emission and both plasma density and electron temperature is much clearer in the SOL than in the edge.

To quantify the correlations, we introduce Spearman's rank correlation coefficient [31], $r_{s}$, which indicates if a monotonically increasing (decreasing) function exists that describes the relation between two quantities, in which case $r_{s}=1\left(r_{s}=-1\right)$. In this case the two quantities are said to be 

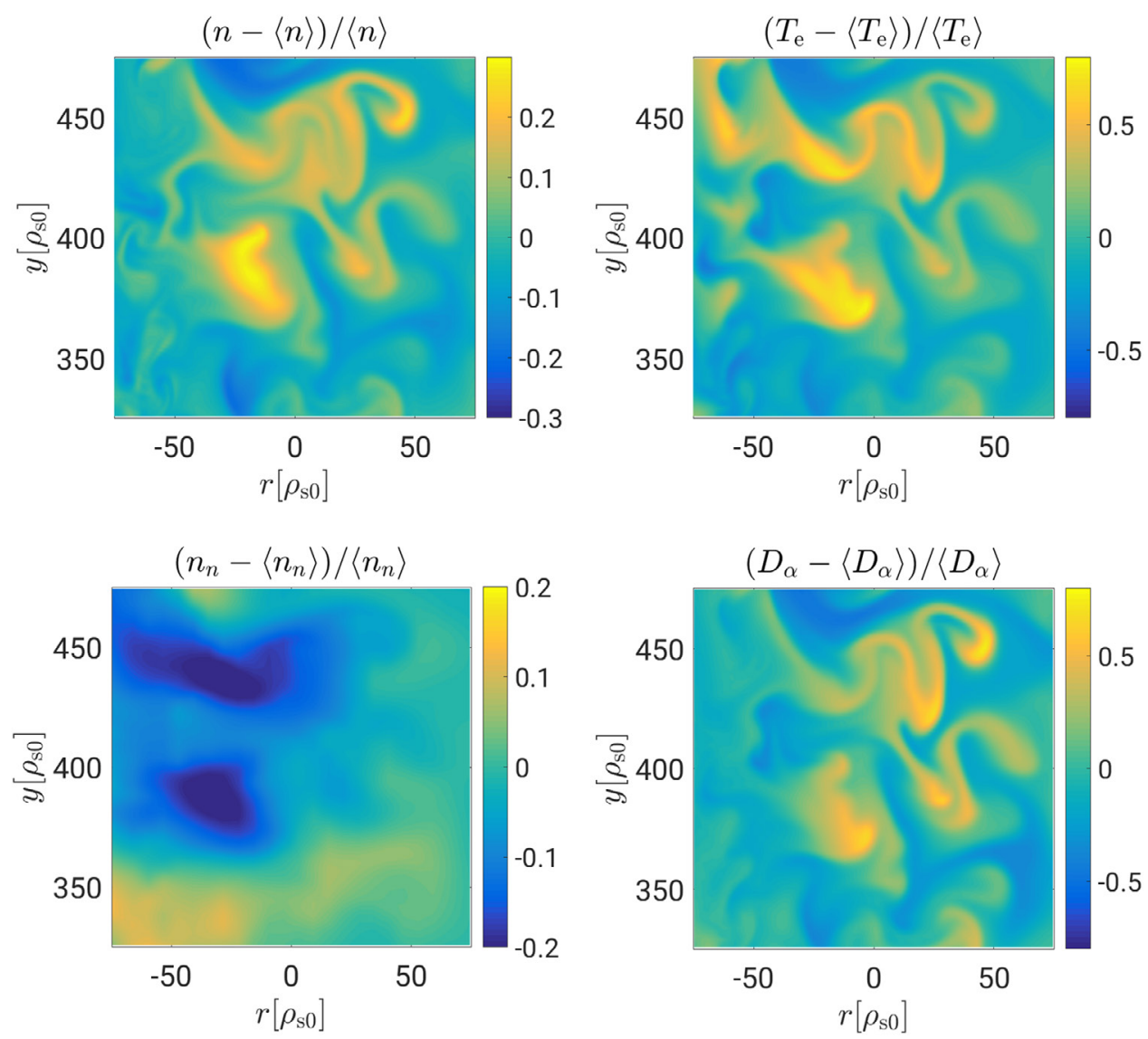

Figure 11. Poloidal snapshots in front of the low-field side gas puff (this region is contoured by a yellow dotted line in figure 3 ) of the normalized fluctuations of plasma density (top left), electron temperature (top right), neutral density (bottom left), and $D_{\alpha}$ emission (bottom right).

correlated (anti-correlated). Spearman's rank correlation coefficient gives the degree of correlation continuously between $r_{s}=1$ and $r_{s}=-1$, and it is independent of the type of correlation (e.g. linear or quadratic). Radial profiles of $r_{s}$ evaluated at the low-field side equatorial midplane are shown in figure 10 for the six combinations of neutral and plasma density, electron temperature, and $D_{\alpha}$ emission. In the SOL, a clear correlation between plasma density, electron temperature, and $D_{\alpha}$ emission is visible, while one observes anti-correlation between the neutral density and the other three quantities. All correlation and anti-correlation coefficients decrease towards the core, and the anti-correlation of neutral density and $D_{\alpha}$ emission in the SOL even turns into a correlation in the confined region. This transition happens at $r \simeq-35 \rho_{\mathrm{s} 0}$ that is, approximately, the radial location inside which the skewness (figure 6, middle panel) and the correlation quantities ( $\tau_{\text {auto }}$, $L_{\text {rad }}$, and $L_{\text {pol }}$, figure 7) differ if they are evaluated from $D_{\alpha}$ or $D_{\alpha\left\langle n_{\mathrm{n}}\right\rangle}$. In fact, for $r \lesssim-35 \rho_{\mathrm{s} 0}$ neutral fluctuations impact not only the $D_{\alpha}$ fluctuation amplitude, but also their spatial structures and fluctuation properties, as it can be observed in the skewness and in the correlation length profiles.

These observations can be explained by the analysis of typical turbulence snapshots. The normalized fluctuations of plasma density, electron temperature, neutral density, and $D_{\alpha}$ emission, in the region in front of the diagnostic gas puff, are shown in figure 11. First, we observe very similar spatial structures in plasma density and electron temperature, confirming that these two quantities are strongly correlated in the whole considered region. Second, for the correlation between the neutrals and the plasma quantities, we distinguish two regions, the region $r \gtrsim-35 \rho_{\mathrm{s} 0}$ (approximately the SOL), which is closer to the outer boundary than the effective neutral mean free path, $\lambda_{\mathrm{mfp}, \mathrm{n}} \simeq 60 \rho_{\mathrm{s} 0}$, and the region $r \lesssim-35 \rho_{\mathrm{s} 0}$, which is further away from the outer boundary than $\lambda_{\mathrm{mfp}, \mathrm{n}}$. In the $r \gtrsim-35 \rho_{\mathrm{s} 0}$ region, positive fluctuations in plasma density and electron temperature (yellow structures) clearly correlate with negative fluctuations in the neutral density (blue structures). This anti-correlation is due to the fact that, while the diagnostic gas puff, the main source of neutral particles at the low-field side midplane, is constant and independent of the local plasma parameters, neutral particles are lost because of ionization processes, which occur with higher probability in regions where the plasma is denser and hotter. Therefore, regions of high plasma density and electron temperature correspond to regions of low neutral density. On the other hand, in the $r \lesssim-35 \rho_{\mathrm{s} 0}$ region, the neutral density is determined not only by the local plasma properties (a weak anti-correlation between $n_{\mathrm{n}}$ and both $n$ and $T_{\mathrm{e}}$ is visible), but also by the plasma properties in the SOL region radially outward from the edge location where the observations are made. In figure 11, the enhanced SOL plasma density and electron temperature, observed at $y \gtrsim 400 \rho_{\mathrm{s} 0}$, reduce the neutral density not only in 
the SOL, but also radially inward. This phenomenon is referred to as shadowing $[5,14]$. On the other hand, for $y \lesssim 350 \rho_{\text {s0 }}$, $n$ and $T_{\mathrm{e}}$ are rather low and let the neutrals penetrate much further than on average. This leads to positive fluctuations in neutral density close to the core, a sort of inverse shadowing event. Because of the non-locality of the shadowing, the neutral density fluctuations in the confined region close to the core are not as anti-correlated with the plasma density and temperature as in the SOL (see figure 10). In fact, the $D_{\alpha}$ emission close to the core is larger when neutrals can penetrate further into the plasma than on average, which is seen in the correlation between $n_{\mathrm{n}}$ and $D_{\alpha}$ in the inner part of the edge region (see figure 10). As a consequence, at a distance larger than $\lambda_{\mathrm{mfp}, \mathrm{n}}$ from the outer boundary, neutral density fluctuations can have a significant influence on the statistical moments and turbulence properties evaluated from $D_{\alpha}$ emission.

We would like to note that we have applied the same synthetic GPI diagnostics to a similar simulation with approximately twice the plasma density, and therefore with approximately half the neutral mean free path, $\lambda_{\mathrm{mfp}, \mathrm{n}} \simeq 30 \rho_{\mathrm{s} 0}$. In this simulation, we observe that the transition from the region where neutrals and $D_{\alpha}$ emission are clearly anti-correlated to the region where the two quantities are correlated, observed in the radial plot of the Spearman correlation coefficients (similar to figure 10), occurs approximately at a distance of $40 \rho_{\mathrm{s} 0}$ from the outer domain boundary. This further confirms that $\lambda_{\mathrm{mfp}, \mathrm{n}}$ is setting the region where the shadowing effect occurs and has a significant impact on GPI measurements.

The relevance of the shadowing effect in interpreting the GPI measurements depends on the experimental set-up and the sensitivity and dynamic range of the cameras. In fact, the location where the shadowing becomes important is related to $\lambda_{\mathrm{mfp}, \mathrm{n}}$, which depends on the experimental conditions. At the same time, the average intensity of the $D_{\alpha}$ emission decreases significantly at distances larger than $\lambda_{\text {mfp,n }}$ (see figure 5, left panel) and therefore it might be recordable only by sensitive cameras with high dynamic range.

\section{Conclusions and outlook}

In this paper, a self-consistent simulation of plasma turbulence and neutral atom dynamics in the SOL and edge regions of a limited tokamak is discussed. This simulation includes two fueling gas puffs on the high-field side and a diagnostic gas puff on the low-field side equatorial midplane. The local $D_{\alpha}$ emission is evaluated and the effect of neutral density fluctuations on GPI measurements is investigated. It turns out that neutral density fluctuations and plasma fluctuations (for both plasma density and electron temperature) are strongly anti-correlated at distances from the gas puff smaller than the neutral mean free path, $\lambda_{\mathrm{mfp}, \mathrm{n}}$, which leads to a systematic influence of neutral density fluctuations on the $D_{\alpha}$ emission amplitude. On the other hand, statistical moments and turbulence characteristics of the $D_{\alpha}$ fluctuations, such as skewness, kurtosis, autocorrelation time, and perpendicular correlation lengths, are not affected significantly in this region, at least in the parameter regime investigated in the presented simulation.
The assumption that one can neglect the impact of neutral fluctuations and interpret the characteristics of the $D_{\alpha}$ emission as being very similar to the characteristics of the plasma, which is often used to interpret experimental GPI measurements, is therefore justified at distances from the gas puff smaller than $\lambda_{\mathrm{mfp}, \mathrm{n}}$. Particular care has to be taken in the analysis of GPI measurements, if regions closer to the core are included in the observations, where the neutrals have traversed distances longer than $\lambda_{\mathrm{mfp}, \mathrm{n}}$ from their source, the diagnostic gas puff. In these regions, in fact, the neutrals have interacted with plasma structures at different radial locations and the $D_{\alpha}$ emission is strongly influenced by non-local shadowing events. This is particularly true for the skewness, kurtosis, autocorrelation time, and radial and poloidal correlation lengths, which are significantly affected by the neutral density fluctuations.

\section{Acknowledgments}

The authors acknowledge F.D. Halpern for providing the correlation diagnostics, and useful discussions with C. Hidalgo, E. de la Cal, and C. Theiler. Part of the simulations presented herein were carried out using the HELIOS supercomputer system at Computational Simulation Centre of International Fusion Energy Research Centre (IFERC-CSC), Aomori, Japan, under the Broader Approach collaboration between Euratom and Japan, implemented by Fusion for Energy and JAEA; and part were carried out at the Swiss National Supercomputing Centre (CSCS) under Project IDs s655 and s718. This research was supported in part by the Swiss National Science Foundation, and has been carried out within the framework of the EUROfusion Consortium and has received funding from the Euratom research and training programme 2014-2018 under grant agreement No. 633053. The views and opinions expressed herein do not necessarily reflect those of the European Commission.

\section{ORCID iDs}

C. Wersal (i) https://orcid.org/0000-0002-3681-3463

\section{References}

[1] Loarte A. et al 2007 Progress in the ITER Physics Basis Chapter 4: power and particle control Nucl. Fusion 47 S203

[2] Lipschultz B. et al 2007 Plasma-surface interaction, scrapeoff layer and divertor physics: implications for ITER Nucl. Fusion 471189

[3] Terry J.L., Maqueda R., Pitcher C.S., Zweben S.J., LaBombard B., Marmar E.S., Pigarov A.Y. and Wurden G. 2001 Visible imaging of turbulence in the SOL of the Alcator C-Mod tokamak J. Nucl. Mater. 290757

[4] Maqueda R.J., Wurden G.A., Zweben S., Roquemore L., Kugel H., Johnson D., Kaye S., Sabbagh S. and Maingi R. 2001 Edge turbulence measurements in NSTX by gas puff imaging Rev. Sci. Instrum. 72931

[5] Zweben S.J. et al 2002 Edge turbulence imaging in the alcator c-mod tokamak Phys. Plasmas 91981

[6] Shesterikov I., Xu Y., Berte M., Dumortier P., Van Schoor M., Vergote M., Schweer B. and Van Oost G. 2013 
Development of the gas-puff imaging diagnostic in the TEXTOR tokamak Rev. Sci. Instrum. 84053501

[7] Zweben S.J., Scott B.D., Terry J.L., LaBombard B., Hughes J.W. and Stotler D.P. 2009 Comparison of scrapeoff layer turbulence in alcator c-mod with three dimensional gyrofluid computations Phys. Plasmas 16082505

[8] Halpern F.D., Terry J.L., Zweben S.J., LaBombard B., Podesta M. and Ricci P. 2015 Comparison of 3D fluxdriven scrape-off layer turbulence simulations with gas-puff imaging of alcator c-mod inner-wall limited discharges Plasma Phys. Control. Fusion 57054005

[9] Janev R.K., Langer W.D., Post D.E. and Evans K. 1987 Elementary Processes in Hydrogen-Helium Plasmas (Berlin: Springer)

[10] Summers H.P., Dickson W.J., OMullane M.G., Badnell N.R., Whiteford A.D., Brooks D.H., Lang J., Loch S.D. and Griffin D.C. 2006 Ionization state, excited populations and emission of impurities in dynamic finite density plasmas: I. The generalized collisional-radiative model for light elements Plasma Phys. Control. Fusion 48263

[11] Moulton D., Marandet Y., Tamain P., Ghendrih P. and Futtersack R. 2014 Density and temperature correlations in the SOL; implications for gas puff imaging of turbulence Contrib. Plasma Phys. 54575

[12] de la Cal E. and The TJ-II Team 2016 Visualising the electron density structure of blobs and studying its possible effect on neutral turbulence Nucl. Fusion $\mathbf{5 6} 106031$

[13] Russell D.A., Myra J.R., D’Ippolito D.A., Munsat T.L., Sechrest Y., Maqueda R.J., Stotler D.P., Zweben S.J. and The NSTX Team 2011 Comparison of scrape-off layer turbulence simulations with experiments using a synthetic gas puff imaging diagnostic Phys. Plasmas 18022306

[14] Stotler D.P., LaBombard B., Terry J.L. and Zweben S.J. 2003 Neutral transport simulations of gas puff imaging experiments J. Nucl. Mater. 313-6 1066

[15] Marandet Y. et al 2011 Transport of neutral particles in turbulent scrape-off layer plasmas Nucl. Fusion 51083035

[16] Mekkaoui A., Marandet Y., Reiter D., Boerner P., Genesio P., Rosato J., Capes H., Koubiti M., Godbert-Mouret L. and Stamm R. 2012 A coarse-grained transport model for neutral particles in turbulent plasmas Phys. Plasmas 19122310

[17] Marandet Y., Tamain P., Futtersack R., Ghendrih P., Bufferand H., Genesio P. and Mekkaoui A. 2013 Influence of neutral particles on scrape-off layer turbulence with application to the interpretation of fast camera data $\mathrm{J}$. Nucl. Mater. 438 S518
[18] Thrysøe A.S., Tophøj L.E.H., Naulin V., Rasmussen J.J., Madsen J. and Nielsen A.H. 2016 The influence of blobs on neutral particles in the scrape-off layer Plasma Phys. Control. Fusion 58044010

[19] Ricci P., Halpern F.D., Jolliet S., Loizu J., Mosetto A., Fasoli A., Furno I. and Theiler C. 2012 Simulation of plasma turbulence in scrape-off layer conditions: the GBS code, simulation results and code validation Plasma Phys. Control. Fusion 54124047

[20] Halpern F.D., Ricci P., Jolliet S., Loizu J., Morales J., Mosetto A., Musil F., Riva F., Tran T.M. and Wersal C. 2016 The gbs code for tokamak scrape-off layer simulations J. Comput. Phys. 315388

[21] Braginskii S.I. 1965 Transport processes in a plasma Rev. Plasma Phys. 1205

[22] Zeiler A., Drake J.F. and Rogers B. 1997 Nonlinear reduced Braginskii equations with ion thermal dynamics in toroidal plasma Phys. Plasmas 42134

[23] Wersal C. and Ricci P. 2015 A first-principles self-consistent model of plasma turbulence and kinetic neutral dynamics in the tokamak scrape-off layer Nucl. Fusion 55123014

[24] Wersal C., Ricci P. and Loizu J. 2017 A comparison between a refined two-point model for the limited tokamak SOL and self-consistent plasma turbulence simulations Plasma Phys. Control. Fusion 59044011

[25] Loizu J., Ricci P., Halpern F.D. and Jolliet S. 2012 Boundary conditions for plasma fluid models at the magnetic presheath entrance Phys. Plasmas 19122307

[26] Arakawa A. 1966 Computational design for long-term numerical integration of the equations of fluid motion: twodimensional incompressible flow. Part I J. Comput. Phys. 1119

[27] Kutta M.W. 1901 Beitrag zur näherungsweisen integration totaler differentialgleichungen Z. Math. Phys. 46435

[28] Riva F., Ricci P., Halpern F.D., Jolliet S., Loizu J. and Mosetto A. 2014 Verification methodology for plasma simulations and application to a scrape-off layer turbulence code Phys. Plasmas 21062301

[29] Halpern F.D. and Ricci P. 2016 Velocity shear, turbulent saturation, and steep plasma gradients in the scrapeoff layer of inner-wall limited tokamaks Nucl. Fusion 57034001

[30] Stangeby P. 2000 The Plasma Boundary of Magnetic Fusion Devices (Bristol: IOP Publishing)

[31] Spearman C. 1904 The proof and measurement of association between two things Am. J. Psychol. 1572 